\title{
ÉVALUER LE SENS DU RISQUE COMMUNICATIONNEL
}

\author{
Thierry De Smedt ${ }^{1}$ \\ avec la collaboration de Lysiane Romain \\ et Odile Remacle, chercheuses ${ }^{2}$
}

\begin{abstract}
The problem is how to evaluate the effects of educational tools intended to develop in young people a capacity «to think the unforeseen of the Net». Evaluating, it is estimating the value of something. Ideally, any evaluation should tend to take into account, in the broadest manner, all dimensions of the thing to be evaluated, because the concept of value returns indisputably to that of totality. No attribution of value can be satisfied by a statement such as "it is well, but...". However, after many confrontations with steps of educational communication, in fields as different as health, the citizenship, the environment, the development or the media culture, it is confirmed as before any globalisation, the evaluation must on the contrary try to be based on restricted dimensions, in particular while following the steps of an média-educational production.

In a landscape of height steps, we concentrate on the specifics stages of the production of educational effects on young people.

Three stages of evaluation are driven: one initial evaluation, an intermediate observation, and a final evaluation, with through one second administration of the initial questionnaire in order to measuring the evolution of the representations of the young people. Talks have also be carried out with participants (professors, educators and parents) to collect their impressions of the course of the activities, like their representations of the
\end{abstract}

Recherches en communication, $\mathrm{n}^{\circ} 22$ (2004). 
integration of those by the young people of their respective groups. This close link between originators and appraisers allows a constant readjustment of the objectives, tools and teaching methods.

The main results of the evaluation concerns

- A globally positive result, producing no exaggerated fears,

- the specific role of teachers, parents and educators,

- the conjunction between the level of use and the Educaunet's productivity

- the typical shape of educational results, characterised by slight improvements,

- the important variance of the results, showing the way for highly flexible educational approaches, adapted to each person and context.

\section{La position du problème}

Comment mesurer les effets de dispositifs éducatifs destinés à développer chez des jeunes une capacité de "penser l'imprévu du net » ? Cette question n'est pas sans enjeux. Notre analyse de ce média nouveau nous le fait apparaitre comme présentant des dimensions originales, rendant l'apparition d'une interaction ou d'une interactivité ${ }^{1}$ plus difficile à anticiper par les jeunes, que lorsqu'ils utilisent les médias traditionnels.

En effet, Internet présente des dimensions nouvelles, si on le compare aux médias traditionnels :

- Abondant : il propose des milliards d'informations et de communication provenant de sources très diversifiées et de qualité inégale,

- Autonome : il procure une liberté de navigation : l'utilisateur déclenche lui-même les requêtes de ses consultations,

- Libéral : il offre peu de contrôle exercé par une autorité,

- Paramétrable : il permet une utilisation solitaire ou entre copains et copines,

- Evolutif : il est en perpétuelle invention de lui-même.

On sait combien, dans de telles circonstances, l'interprétation des messages médiatiques se réfère inévitablement à des éléments contextuels. Or, Internet est 
- un espace de communication décontextualisé : chaque site, chaque page même, se réfère à un contexte particulier, généralement indépendant de la navigation de l'usager,

- un espace indifférencié de communications différenciées,

- un espace de communication non transparent : il est assez aisé de diffuser des informations sur Internet en dissimulant son identité à ses interlocuteurs.

Il est par conséquent nécessaire de mettre en place des outils éducatifs capables de développer les compétences communicationnelles des jeunes utilisateurs d'Internet en matière d'interprétation critique des documents qu'ils y trouvent et échangent. La question qui nous occupera ici est de définir les méthodes aptes à évaluer de tels outils.

\section{Évaluer une production en éducation aux médias. Une approche segmentée}

Evaluer, c'est construire un jugement sur la valeur de quelque chose. Idéalement, toute évaluation devrait tendre à prendre en compte, de la manière la plus large, toutes les dimensions de la chose à évaluer, car la notion de valeur renvoie indiscutablement à celle de totalité, ou au minimum, de complexité. Aucune attribution de valeur ne peut se satisfaire d'un énoncé aussi vague que « c'est bien, mais, ... ». Or, après de nombreuses confrontations à des démarches de communication éducative, dans des domaines aussi différents que la santé, la citoyenneté, l'environnement, le développement ou la culture médiatique, il se confirme qu'avant toute globalisation, l'évaluation a intérêt à se fonder sur des dimensions restreintes, notamment en suivant les étapes d'une production médio-éducative.

C'est une manière de mieux cerner les moments critiques de choix et d'action, en évitant de mettre en cause l'ensemble du processus. Ainsi les indications issues de l'évaluation permettent-elles d'agir, à des fins d'optimisation, sur des segments restreints de la démarche éducative, tout au long de la production de la méthode et des outils, sans provoquer de crise globale. Nous allons aborder cette question à travers le cas concret du projet Educaunet. 


\section{Approche segmentée de la production Educaunet}

La production de la méthode et des outils Educaunet peut être schématisée sous la forme de huit segments, applicable à d'autres démarches éducatives analogues, présentant les étapes d'un modèle général. Décrivons-les brièvement.

Au départ, il existe un état initial de la réalité, ici, l'avènement d'Internet et son usage libre par les jeunes de plus en plus nombreux. Cette réalité est vue par certains, comme porteuse de dangers, au point de conduire la Commission européenne à lancer un appel d'offre, en 1999 , en vue d'un «Internet plus sûr pour les jeunes $»^{2}$, dont la réponse attendue relevait essentiellement de techniques de surveillance et de filtrage. Ce couplage entre une situation réelle et sa problématisation est le premier axe du schéma segmenté.

Le consortium initial, constitué par le Clemi, Média-Animation et le GreMS a réagi à cette problématisation en proposant une approche éducative (second segment), inspirée de l'éducation aux médias. Elle consistait à aider des parents, des éducateurs et des enseignants à favoriser chez les jeunes une meilleure compréhension de la réalité d'Internet, dans toutes ses dimensions médiatiques et non seulement technique, comme la majorité des quelques formations existantes, en développant une relation meilleure avec Internet (segment trois).

Schématiquement, les trois segments principaux peuvent être représentés par la figure suivante.

Cette première schématisation mérite d'être approfondie au moyen d'une complexification du segment deux, à savoir le processus éducatif. Celui-ci repose sur un modèle précis, issu de l'expérience en éducation aux médias, des trois membres du consortium.

L'hypothèse était qu'ainsi les jeunes seraient mieux équipés pour s'approprier des usages plus sûrs d'Internet, à la fois plus curieux et plus perspicaces, s'ils bénéficiaient d'un accompagnement d'adultes, à travers des activités d'explorations, de communication, d'information et de prise de conscience de leur situation d'usagers. Lors de la définition des activités à expérimenter, celles-ci ont en effet été réparties en cinq groupes recouvrant les différentes attitudes opératoires de l'usager 


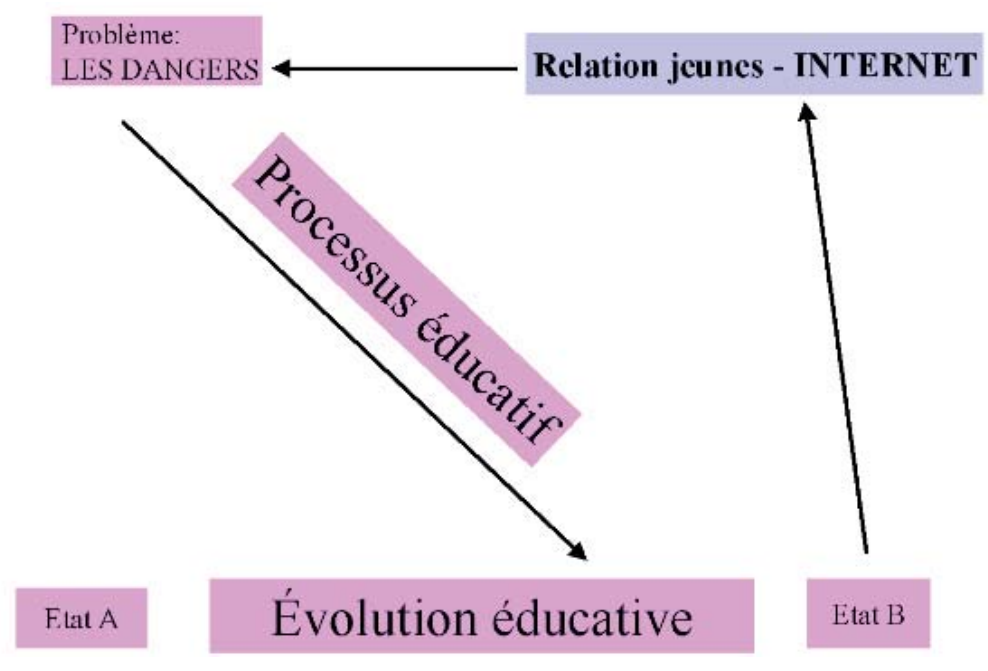

Changement de représentation, d'attitude, de comportement auprès d'un public

d'Internet : explorer, qui s'adresse plutôt aux débutants, s'informer, interagir et s'exprimer qui traitent de l'information, de la communication et de la création, et enfin s'observer qui invite à prendre du recul par rapport à ses pratiques d'Internet.

Par conséquent, le segment deux se subdivise, après approfondissement, en six segments distincts.

Le premier (segment numéro deux, à présent) concerne la définition d'une stratégie éducative, spécifiant les bases de l'approche, en l'occurrence la distinction entre danger et risque, et les destinataires - relais et finaux - de l'éducation. Ensuite vient le segment trois, consistant à connaître au mieux les caractéristiques des destinataires de l'éducation : les jeunes, mais aussi leurs parents, enseignants et éducateurs.

Ces données permettent de concevoir une méthode adéquate, adapté à la fois aux objectifs et aux publics (segment quatre).

Cette méthode guide la réalisation d'outils éducatifs (segment cinq)

Les outils ainsi créés doivent parvenir à leurs destinataires, désignés par le vocable usuel d'audience (segment six). 
Vient alors l'étape cruciale de la production, auprès de l'audience des effets éducatifs souhaités, caractérisés par des changements de représentations, d'attitudes et de comportement (segment sept), basés sur une meilleure compétence dans la prise de risque.

Au total le schéma compte huit segments de production, dont les plus centraux sont au coeur de la démarche de communication éducative. Cela donne le schéma suivant.

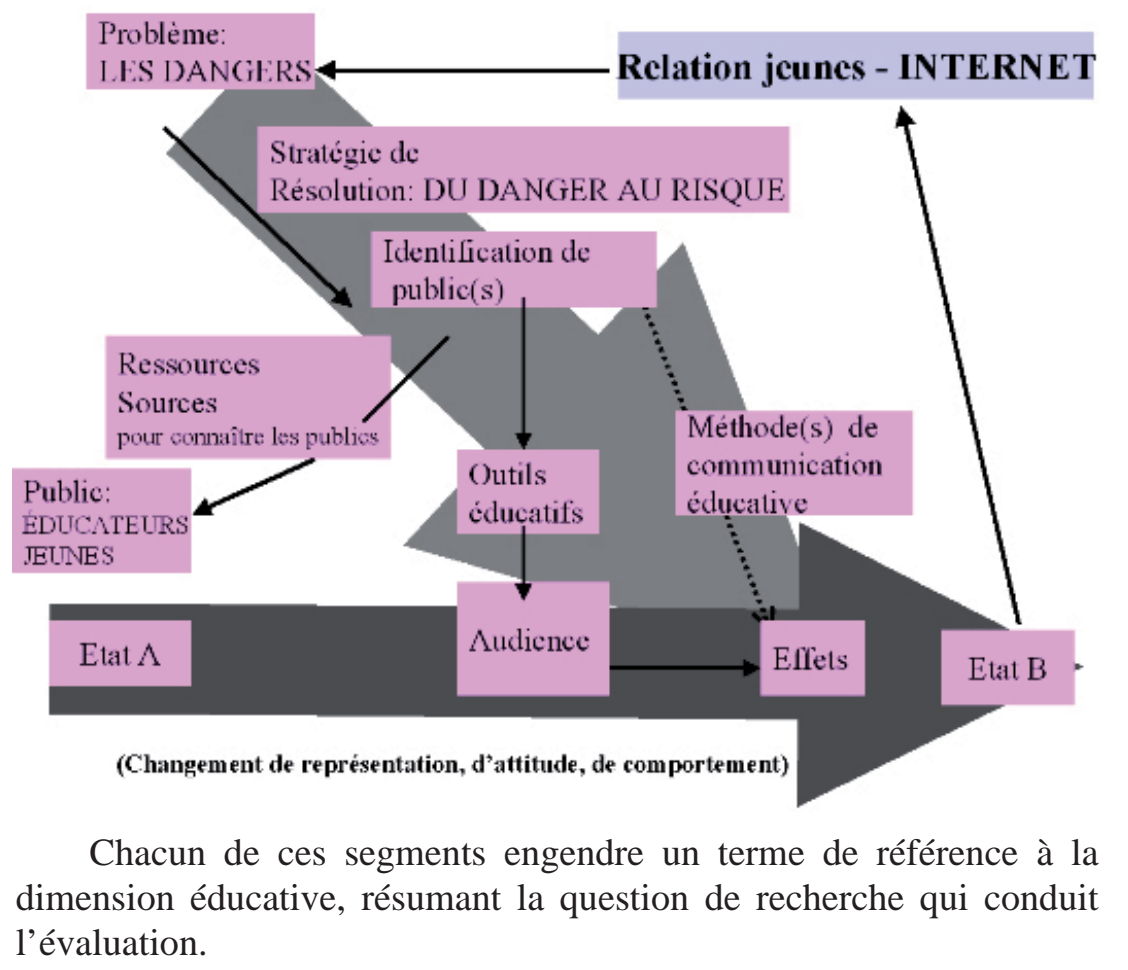




\begin{tabular}{|c|c|c|}
\hline & Description & Question d'évaluation \\
\hline 1. & $\begin{array}{l}\text { Problématiser la relation Jeunes et } \\
\text { Internet }\end{array}$ & $\begin{array}{l}\text { Le problème identifié est-il encore } \\
\text { prégnant? }\end{array}$ \\
\hline 2. & $\begin{array}{l}\text { Elaborer une stratégie de résolution } \\
\text { distinguant danger et risque }\end{array}$ & $\begin{array}{l}\text { Notre public est-il réellement concerné } \\
\text { par le problème? }\end{array}$ \\
\hline 3. & $\begin{array}{l}\text { Connaître au mieux les caractéris- } \\
\text { tiques des destinataires de l'éduca- } \\
\text { tion }\end{array}$ & $\begin{array}{l}\text { Notre connaissance du public est-elle à } \\
\text { jour? }\end{array}$ \\
\hline 4. & $\begin{array}{l}\text { Concevoir une méthode adéquate } \\
\text { adaptée aux objectifs et aux publics }\end{array}$ & $\begin{array}{l}\text { La méthode est-elle susceptible de } \\
\text { produire l'effet escompté, auprès du } \\
\text { public destinataire? }\end{array}$ \\
\hline 5. & Réaliser les outils éducatifs & Les outils sont-ils efficaces? \\
\hline 6. & $\begin{array}{l}\text { Acheminer les outils vers leur } \\
\text { audience }\end{array}$ & Les outils atteignent-ils le public visé? \\
\hline 7. & $\begin{array}{l}\text { Engendrer des changements de } \\
\text { représentations d'attitude et de } \\
\text { comportement }\end{array}$ & $\begin{array}{l}\text { Les outils produisent-ils les changements } \\
\text { escomptés? }\end{array}$ \\
\hline 8. & $\begin{array}{l}\text { Modifier la relation des jeunes à } \\
\text { Internet }\end{array}$ & $\begin{array}{l}\text { Les changements opérés sur le public indui- } \\
\text { sent-ils une modification sur la relation } \\
\text { jeunes-Internet, dans le sens d'une réduc- } \\
\text { tion du problème initialement identifié? }\end{array}$ \\
\hline
\end{tabular}

Voici comment se situent les question, dans le shéma des huit segments de la production.

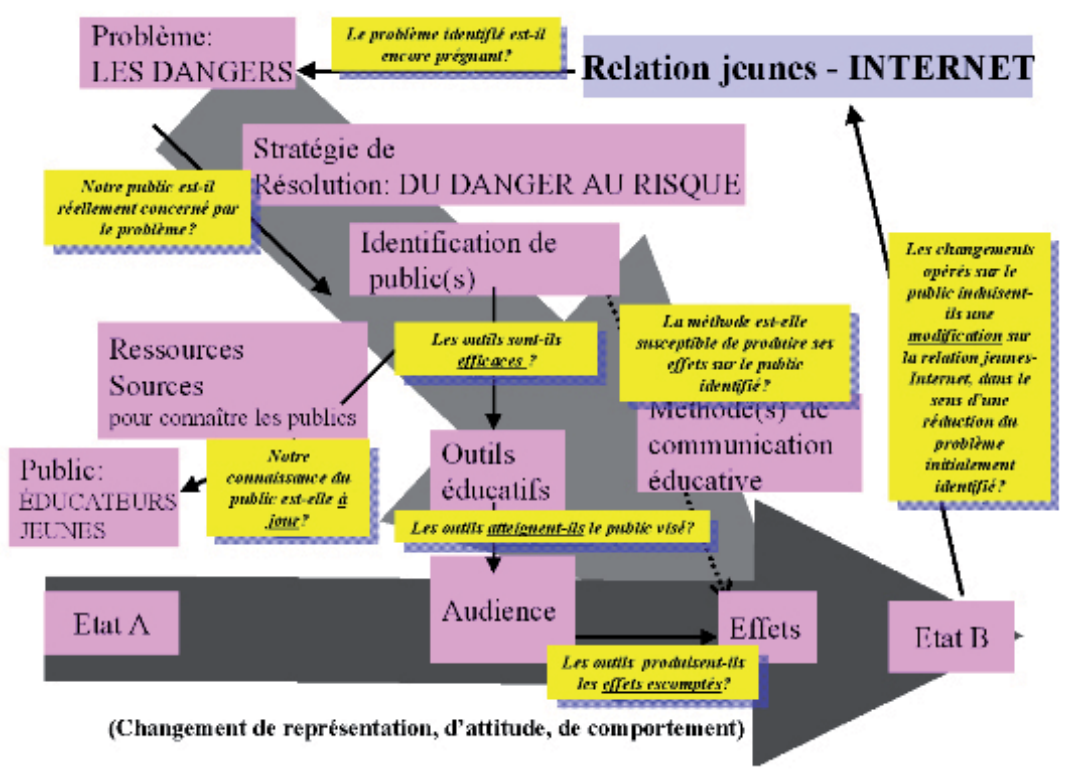


Notre propos ne sera pas de couvrir l'ensemble de l'évaluation de la démarche de production d'Educaunet. Nous nous limiterons à la question initiale : quelle est la valeur des effets éducatifs produits par la méthode et les outils Educaunet. En somme notre examen se limitera à la zone claire, dans le schéma ci-dessous : celle qui tente de repérer en quoi les jeunes ayant pratiqué les activités Educaunet évoluent dans leurs représentations et leurs attitudes d'usagers d'Internet.

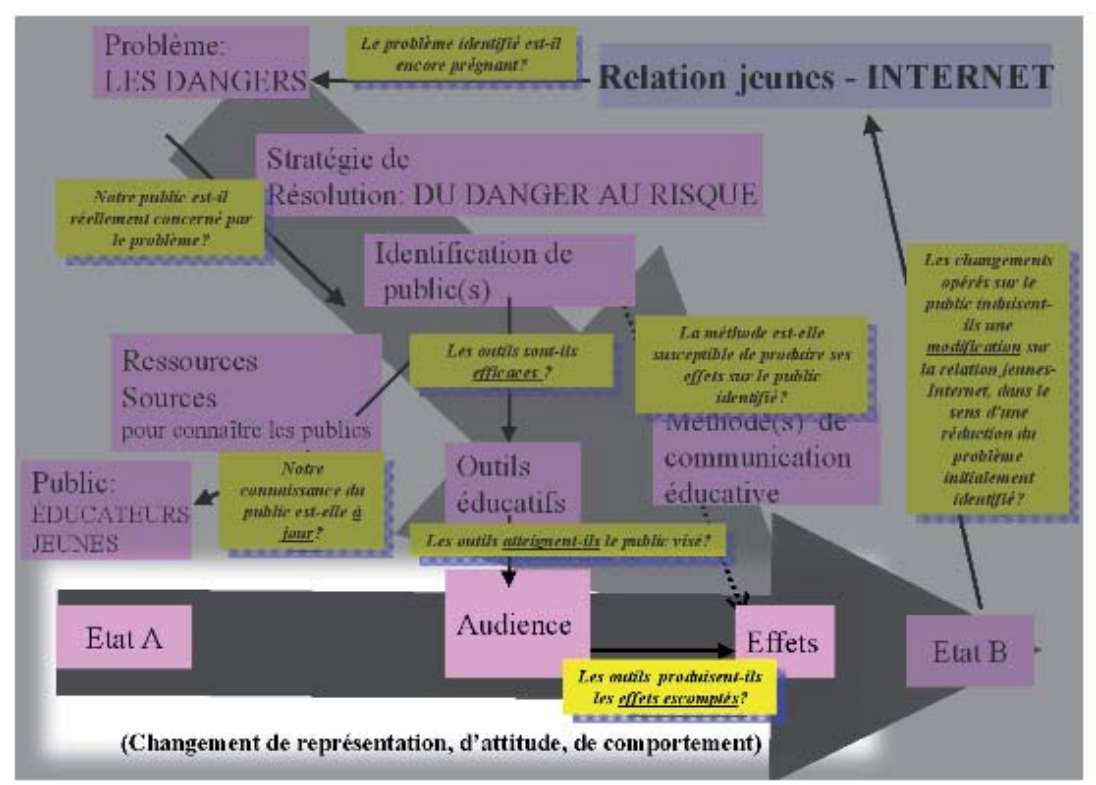

\section{Méthodes d'investigation}

Dans un premier temps, nous allons décrire la manière dont ont été effectuées les évaluations visant à valider et optimiser la version expérimentale de la méthode et des outils Educaunet, réalisée en 2001, dans la phase de production initiale de l'outil.

Une observation préalable des usages d'Internet a été réalisée sous l'intitulé "Internet et les jeunes $»^{3}$. A l'initiative de partenaires canadiens ${ }^{4}$, cette étude à été menée parallèlement dans plusieurs pays ${ }^{5}$. Cette recherche est décrite, dans ce numéro, par Isabelle Bréda. Elle aborde en effet de manière privilégiée et approfondie les usages des jeunes de 12 à 17 ans vis-à-vis d'Internet et constitue ainsi une première photographie d'un processus en pleine évolution. 
«Internet et les jeunes » visait également à apporter un éclairage sur la façon dont les jeunes perçoivent la sécurité sur Internet. Volet que nous avons largement approfondi dans l'enquête menée au sein d'Educaunet, et dont la finalité est l'analyse de l'évolution des représentations que se font les jeunes des risques liés à Internet. Cette approche a permis d'établir quelques points de départs sur la manière dont les jeunes voient les risques liés à Internet en France et en Belgique. Les résultats obtenus dans ces deux pays sont très similaires, ce qui nous a conduit à ne pas les différencier dans le protocole d'évaluation mis en place dans le projet Educaunet.

«A priori, les jeunes ne situent pas Internet du côté du danger, mais l'envisage de manière positive. C'est seulement une fois sollicités sur cette question que les jeunes citent des risques. Ceux-ci sont alors de différentes natures:

- L'appauvrissement du contact social, l'isolement, la solitude, l'exclusion des plus démunis et la pauvreté ;

- Les virus;

- Le vol et le banditisme;

- Les sites racistes, pornographiques et pédophiliques (bien que ce genre de sites soient relativisés par les jeunes. Le risque leur paraît réel mais confiné)

- La dissimulation et le mensonge (Il est intéressant de constater à ce sujet que l'on retrouve les mêmes mobiles que ceux qui concerne la propagation de rumeurs ${ }^{6}$ : «c'est le fait de "ceux qui ne travaillent pas», les "privés». Les jeunes de 17-18 ans en sont menacés!»

- Enfin, la généralisation du mode de communication superficiel au détriment de la rencontre et de la communication face-à-face. «Rien ne remplace la présence réelle». »7

«La plupart des jeunes ont entendu parler de dangers liés à Internet, mais rares sont ceux qui y ont été confrontés directement. Le plus souvent, leur perception d'un danger provient des discours médiatiques et parentaux et la crainte qu'ils éprouvent est d'autant plus grande qu'ils connaissent mal Internet : moins les jeunes l'utilisent et moins ils lui font confiance. ${ }^{8}$

«En synthèse, on peut considérer que la relation des jeunes aux risques d'Internet présente les caractéristiques suivantes: 
- ils sont rarement évoqués spontanément par les jeunes, et ne sont pas l'objet d'une préoccupation explicite ;

- ils deviennent volontiers l'objet d'intérêt et de réflexion lorsque le jeune est sollicité à ce propos ;

- ils sont l'objet de demande d'informations lorsque le jeune est sensibilisé ;

- ils semblent refléter assez fidèlement le contexte de problématisation dans lequel le jeune se trouve immergé(risques techniques, idéologiques, sociaux, etc.). ${ }^{9}$

Par conséquent, l'équipe de recherche et de conception d'Educaunet s'est donnée comme moyen éducatif le développement, chez les jeunes, d'une attitude de clairvoyance face au danger, basée sur une vision réaliste des risques possibles (pour soi et pour autrui) consécutifs à une action d'utilisateur (consulter, télécharger, publier, solliciter, etc)

\section{Modalités de l'évaluation}

\section{Structure des groupes tests}

Trois types différents de personnes-relais ont été choisis pour participer au projet Educaunet : des enseignants, des éducateurs de mouvements associatifs et des parents. Ces personnes-relais sont les médiateurs du projet auprès des jeunes avec qui elles sont en contact. Après avoir reçu une formation les 5 et 6 octobre 2000, les personnesrelais ont en charge l'animation de certaines activités, choisies dans le coffret mis à leur disposition, auprès de leur public de jeunes. Le cas des parents est légèrement différent. L'évaluation se base sur l'analyse et l'expérimentation des activités pratiquées au sein même d'un groupe de parents.

Les groupes ont été ainsi répartis :

- 6 professeurs d'enseignement primaire (3 en France et 3 en Belgique)

- 12 professeurs d'enseignement secondaire (6 en France et 6 en Belgique)

- 4 « parents-relais » ( 2 en France et 2 en Belgique)

- 7 animateurs socio-culturels (4 en France et 3 en Belgique) 
Trois étapes chronologiques d'évaluation sont mises en œuvre :

Une évaluation initiale. Elle consiste administrer des questionnaires aux jeunes qui participent au projet. Ce questionnaire vise à saisir leur manière d'envisager les dangers liés à Internet. Un questionnaire identique sera également distribué au début et à la fin de chaque phase expérimentale. Ce même processus est également mis en place via les associations de parents.

Une évaluation intermédiaire. Celle-ci passe par l'observation directe du déroulement des activités dans les différents groupes tests. Après chaque activité, les participants sont invités à remplir des fiches d'évaluation. Ceci permet de recueillir des informations sur le déroulement de l'activité, même lorsque les observations directes ne sont pas possibles.

Une évaluation finale, au travers une seconde administration du questionnaire initial afin de mesurer l'évolution des représentations des jeunes. Mais aussi grâce à des entretiens individuels plus approfondis avec certains jeunes ayant suivi la formation. Des entretiens seront également effectués avec certains participants (professeurs, animateurs et parents) pour recueillir leurs impressions du déroulement des activités, ainsi que leurs représentations de l'intégration de celles-ci par les jeunes de leurs groupes respectifs.

La démarche d'Educaunet combine la conception et l'évaluation, c'est pourquoi les concepteurs des outils éducatifs participent euxmêmes au processus d'évaluation, tout comme l'équipe d'évaluation participe à la conception. Ce lien étroit entre concepteurs et évaluateurs permet un réajustement constant des objectifs, outils et méthode de formation. Pour répondre à cette volonté de travail en collaboration constante, plusieurs réunions ont été organisées entre les concepteurs et les évaluateurs du projet pour élaborer les activités Educaunet. 


\section{Tableau synoptique des étapes de l'évaluation}

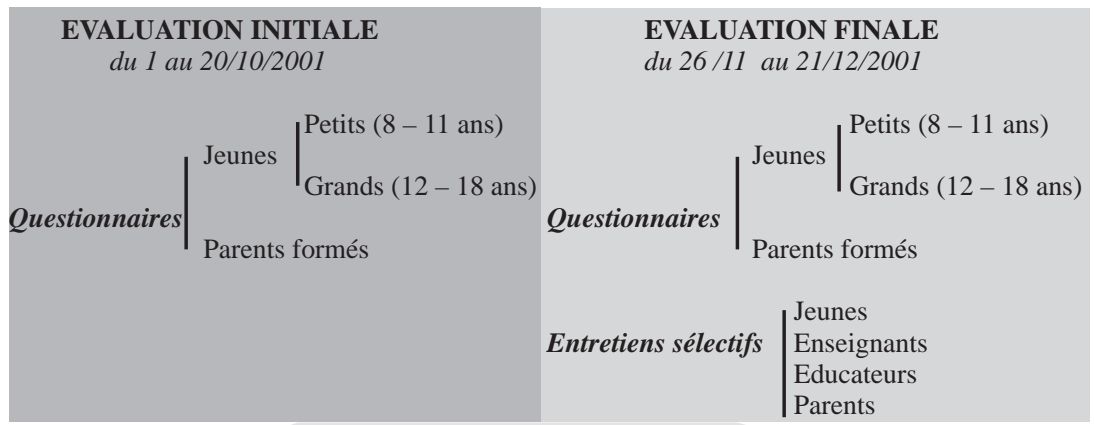

EVALUATION INTERMEDIAIRE

du 20/10 au 1/12/2001

$\begin{array}{ll}\text { Activité } & \begin{array}{c}\text { Fiche d'évaluation par les participants } \\ \text { (différente pour les parents) }\end{array} \\ \text { Observations directes } & \text { par les chercheurs (sauf parents) }\end{array}$

\section{L'évaluation initiale : les questionnaires}

L'objectif premier des questionnaires est de permettre à l'équipe d'Educaunet d'établir un état des lieux des représentations que se font les jeunes ainsi que les parents des risques liés à l'utilisation d'Internet. Cette étape doit permettre de mesurer l'impact de la formation sur les représentations des jeunes.

En outre, cette étape permet d'identifier des profils et de constituer des groupes.

L'élaboration des questionnaires a consisté en une reformulation plus concrète des hypothèses construites au cours du séminaire de problématisation. Repartant du tableau récapitulatif couvrant l'ensemble des risques évoqués, nous l'avons traduit en questions compréhensibles en fonction des différences d'âges des jeunes concernés. Une partie succincte est également consacrée aux pratiques actuelles et à l'utilisation d'Internet chez les jeunes. Nous avons en effet souhaité privilégier les représentations liées aux risques d'Internet, volet qui exigeait les approfondissements nécessaires. 
Deux types de questionnaires ont été administrés aux différents publics choisis : les enfants de 8 à 11 ans, les jeunes de 12 à 18 ans. Le premier questionnaire, constitué de quatre pages, reprend, dans un langage simplifié, les mêmes questions liées aux risques d'Internet. Le second comprend huit pages de questions de compréhension, à destination des jeunes entre 12 et 18 ans ainsi que des parents.

Certains parents ont en effet été sollicités en vue de mesurer les éventuelles convergences et divergences d'opinion. Il leur a donc été présenté le même questionnaire que celui qui était destiné aux jeunes de $12-18$ ans.

Quant à l'administration des questionnaires, elle a été systématique en ce qui concerne chaque classe participant à la phase d'expérimentation qui a fait ensuite l'objet d'un temps d'observation. Cela représente environ mille questionnaires complétés par les jeunes eux-mêmes.

Pour chaque nouveau groupe-test, nous avons restreint nos observations à quelques activités. Le choix de celles-ci s'est effectué sur base de leur sphère d'appartenance. Nous avons opté pour l'observation de deux activités par sphère dans chaque groupe-test.

Voici un tableau synoptique représentant les 4 nouveaux groupes élaborés pour la phase d'observation des activités :

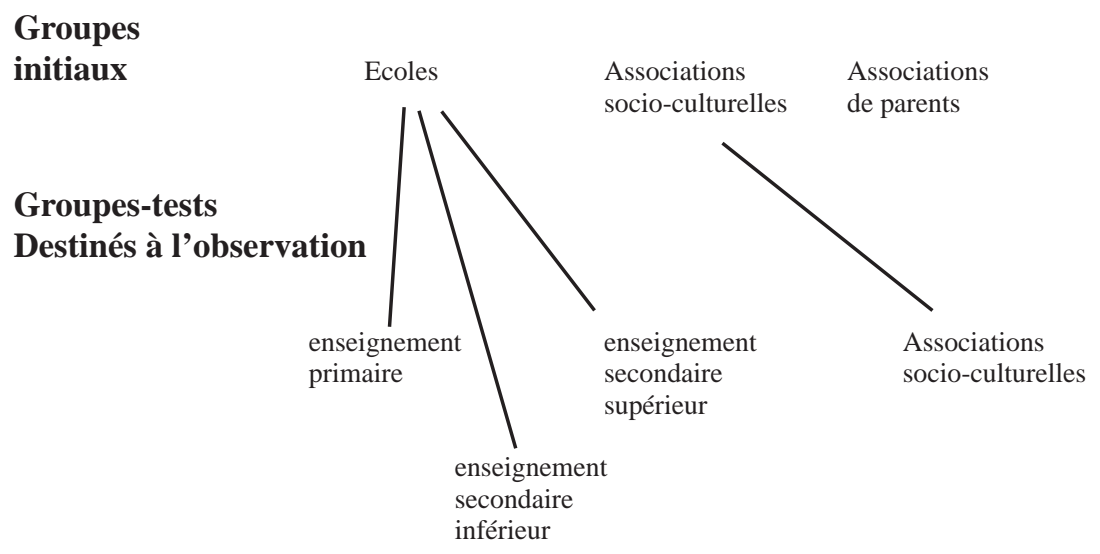




\section{L'évaluation finale : les questionnaires et les entretiens}

Comme nous l'avons déjà indiqué, lors de l'étape finale de l'évaluation de chaque phase expérimentale, nous redistribuerons aux jeunes et aux parents le même questionnaire que celui de l'étape initiale d'évaluation. Ceci vise à analyse l'évolution des représentations des dangers liés à Internet après avoir participé aux activités Educaunet. Notre hypothèse de départ étant que la participation aux activités Educaunet permet une conscientisation des jeunes des dangers liés à Internet.

Parallèlement, des entretiens plus approfondis seront menés auprès d'un échantillon de jeunes. Ces entretiens se dérouleront selon deux volets. Le premier s'attachera à la formation suivie. Il visera à recueillir les impressions des jeunes quant au déroulement des activités, à leur bien-fondé, etc. Le second volet touchera plus particulièrement les représentations des jeunes par rapport aux dangers d'Internet, tout comme dans les questionnaires écrits. Ceci permettra de mieux comprendre la formation de ces représentations.

\section{Les résultats marquants}

Nous nous limiterons aux grandes tendances des résultats.

L'évaluation procure globalement une confirmation de l'efficacité $\mathrm{d}^{\prime} E d u c a u n \mathrm{t}^{10}$ : les éducateurs et les jeunes manifestent un intérêt pour la méthode et les outils et se les approprient. Parmi les aspects remarquables apportés par l'évaluation, on peut signaler les points suivants.

\section{L'action sur les éducateurs : des effets différenciés}

Parmi les trois publics distincts auxquels s'adresse Educaunet, (parents, éducateurs, enseignants) chacun constitue une réalité différente à laquelle il s'agit d'adapter l'approche. Dans familles et le milieu associatif, les résultats relèvent avant tout de la découverte. L'interrogation première des parents est de savoir ce que peuvent aller chercher les jeunes sur Internet et ce qu'ils y trouvent en réalité. L'évaluation montre qu'en général, les parents saisissent mieux le potentiel d'Internet et perçoivent ses contraintes, voire ses dangers, de manière plus réaliste. Les éducateurs associatifs se préoccupent davantage de la réussite d'activités impliquant une bonne participation des jeunes. Ils attachent ainsi 
beaucoup d'importance à la qualité sociale des activités et leur impact sur la vie collective du groupe.

Dans l'enseignement, la vision d'Internet est plus utilitaire : on y cherche un moyen d'enseigner. Les enseignants attendent de l'usage d'Internet une efficacité en documentation et le développement, chez l'élève, de capacités expressives. Le contexte scolaire et le milieu associatif offrent un cadre structurant pour la réalisation d'activités de discussion et de débat. Les parents diffusent davantage les messages éducatifs à travers des les moments d'échanges épisodiques et informels, en alternance avec les activités éducatives. Ces trois agents-relais éducatifs semblent donc trouver dans les activités proposées les matériaux qu'ils recherchent et les choisissent à des fins spécifiques. Plus encore, les enseignants saisissent souvent la structure des activités et développent des variantes sur le même modèle, étendant ainsi la portée des outils en les accommodants.

\section{Les effets sur les jeunes}

Concernant les jeunes, on peut distinguer deux fonctions essentielles.

Une fonction initiatrice. On remarque que ce sont majoritairement les jeunes qui se disent «non utilisateurs » d'Internet qui manifestent une évolution croissante dans leur prise de conscience des risques. Educaunet initie les néophytes et leur permet de découvrir des réalités dont ils ne se doutaient pas.

Quant aux utilisateurs plus réguliers, nous observons une plus grande évolution dans les représentations des utilisateurs réguliers en termes de précision de la vision des risques. Ces changements de représentation se manifestant essentiellement lorsque les jeunes affrontent les problématiques les plus complexes du risque, telles que la séduction, le travestissement de l'identité, la possibilité de faire du mal à autrui.

L'usage réel d'Internet par les jeunes reste donc un facteur important de son appropriation en termes de risques, se conjuguant avec Educaunet, sans que l'un soit le substitut de l'autre.

Cette différence due au degré d'usage, dans les effets éducatifs produits, renforce l'idée que le phénomène Internet, pour collectif qu'il soit, reste lié aux personnes : chaque jeune possède un profil propre, 
un type d'utilisation et des représentations personnelles : la variance des résultats est élevée. Cela plaide pour des approches différenciées, adaptées à chacun. En revanche, la confrontation et l'échange des expériences, comme les induisent les activités Educaunet, encouragent les jeunes à ouvrir leurs représentations aux expériences vécues par les autres.

Les évolutions constatées ne se font jamais sous une forme discontinue, passant du tout au rien. Le plus souvent, les variations dans les réponses aux questionnaires sont de l'ordre de cinq à vingt pour cent, dans le sens attendu. Rarement plus. Cela confirme la métaphore d'une action éducative douce, mais cumulative, bien éloignée de l'image du vaccin qui prévaut encore souvent dans l'imaginaire de la protection.

Qualitativement, une similitude remarquable est apparue, à propose de l'évolution de la connaissance par les jeunes des risques d'Internet, entre l'observation faite en France et en Belgique en 2001 et celle menée durant Educaunet, phase 2, par Andrew Burn et Rebekah Willett, de l'Université de Londres ${ }^{11}$ : Dans les deux cas, avant Educaunet, les jeunes citent des risques irrationnels, par exemple, apprendre à fabriquer des bombes, les «black mail» (courriers nocif), des gens «qui prennent les commandes de mon ordinateur », un enlèvement. Ils expriment aussi des confusions dans la nature des risques, par exemple en redoutant des demandeurs d'asile, la propagation du Sida, l'usage de drogues apportées par Internet, les extra terrestres ou le danger de devenir aveugle face à l'écran.

Après Educaunet, la gamme des risques évoqués est plus étroite, mais plus réaliste et plus précise : perte de temps, coûts cachés, publicités trompeuses, sont des risques qui sont cités plus souvent.

Ce gain de rationalité apparaît particulièrement bien au travers d'une méthode originale, bien maîtrisée par l'équipe de Londres, basée sur la production par les jeunes de cartes de réseaux conceptuels. À titre d'exemple, voici la carte des risques dessinée par un enfant de dix ans, avant Educaunet. 


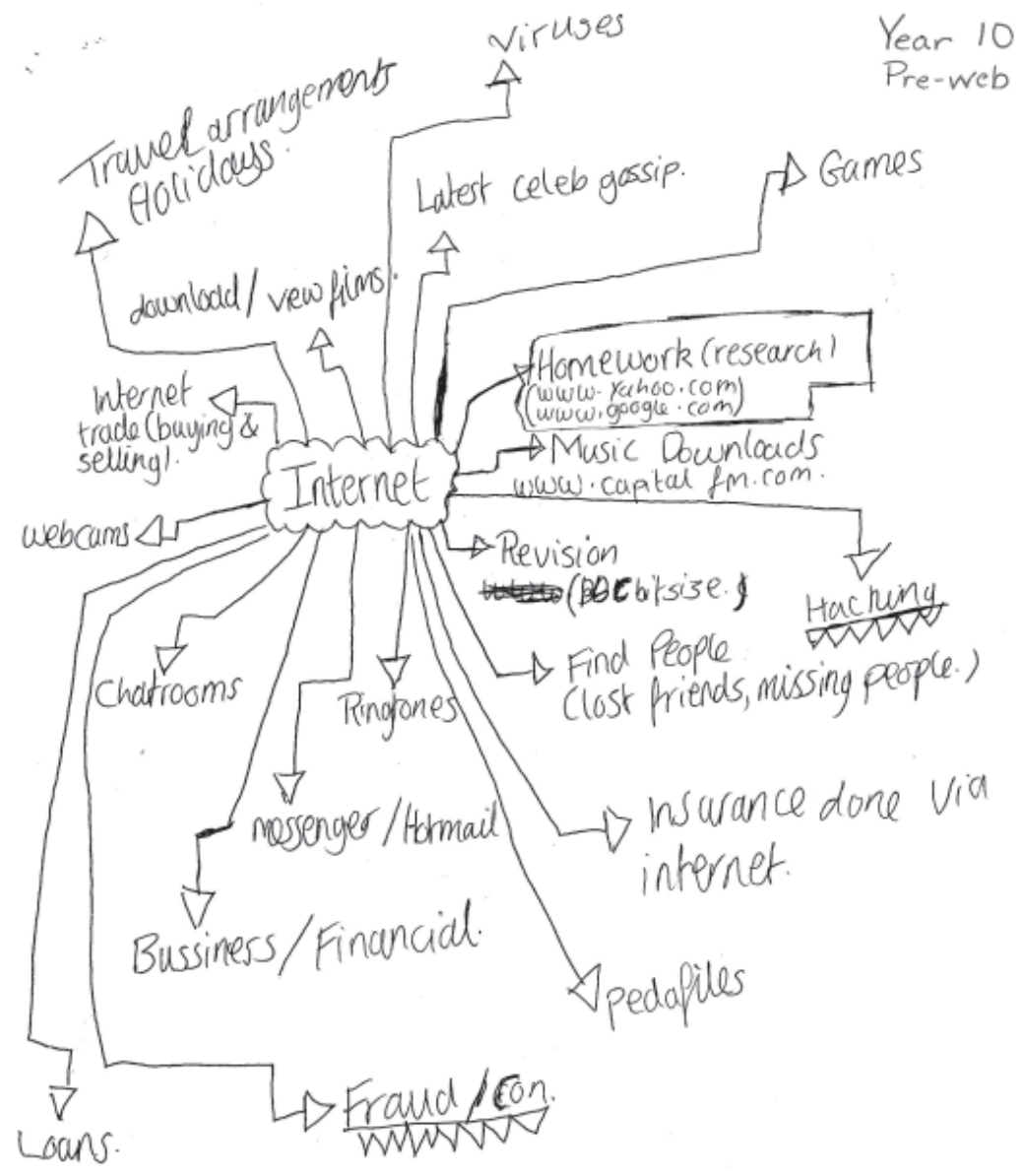

(Source : évaluation effectuée par Andrew Burn (Lecturer), Rebekah Willett (Research Officer), University of London, Educaunet 2.) 
Après les activités Educaunet en classe, à la même demande, le dessin de la carte devient

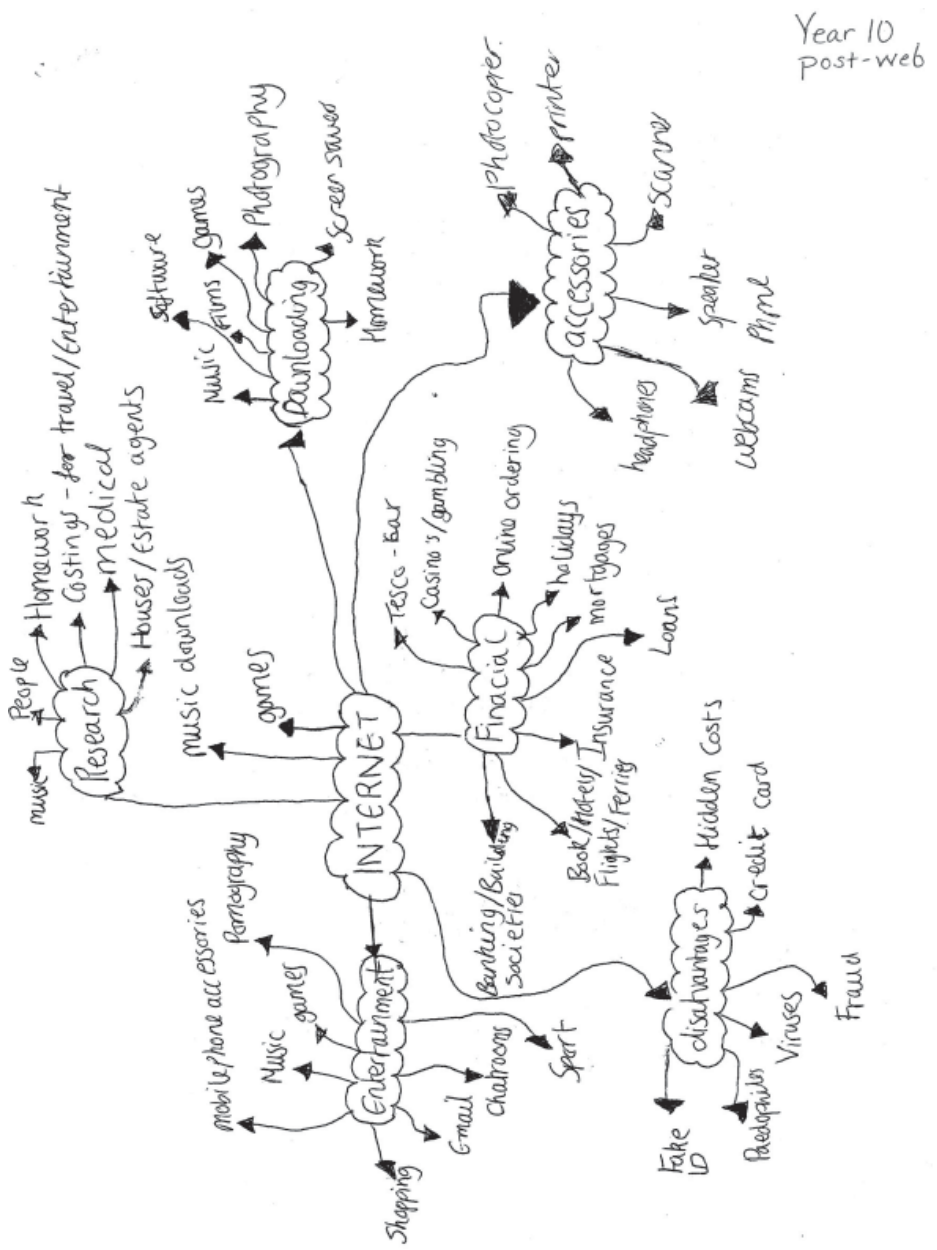

(Source : Evaluation réalisée par Andrew Burn (Lecturer), Rebekah Willett (Research Officer), University of London, Educaunet 2.)

On le voit, le réseau comprend davantage de niveaux, groupés par thèmes et organisés logiquement aussi bien que techniquement : Internet au centre, reliéà « Recherche », « Téléchargement », « Divertissement », « Finances », « Accessoires » et «Inconvénients ». 
Les différences entre les deux dessins expriment bien l'évolution en précision et en organisation de la pensée des jeunes, face aux risques d'Internet. En outre la présence et l'importance d'éléments positifs dans des deux dessins prouvent que le jeune ne s'est pas focalisé sur les risques, mais garde sa capacité de voir en Internet comme un phénomène positif.

\section{Bilan}

A la question «comment évaluer le sen du risque », la démarche décrite dans cet article suggère essentiellement d'aborder la question sous un angle différentiel, en comparant des situations initiales et finales. La difficulté majeure réside dans le fait que le sens du risque n'est détectable que dans des situations concrètes qui peuvent avoir une force déterminante sur l'activation du sens du risque. Cela suggère

- d'adopter le plus large éventail d'investigations possibles, afin d'en saisir les multiples dimensions (techniques, relationnelles, cognitives, affectives, ...),

- d'accorder de l'attention à l'environnement des jeunes (parents, éducateurs, cadre institutionnel scolaire ou familial, amis, ...), car ces contextes influencent l'attitude du jeune face au rique,

- de rester ouvert à détecter des compétences mêmes latentes, susceptibles de s'épanouir rapidement dans le contexte de nouveaux usages d'Internet par le jeune, car celui-ci évolue constamment dans ses pratiques. Internet est pour lui un véritable terrain d'entraînement.

\section{Notes}

1 Selon Geneviève JACQUINOT-DELAUNAY et Monique LINARD, on peut distinguer INTERACTIVITE - Interagir avec la machine

L'interactivité est en revanche définie de la façon suivante : "Propriété des médias, des programmes et des systèmes liés de façon plus ou moins constitutive à un ordinateur de pouvoir entretenir un dialogue plus ou moins poussé avec l'utilisateur. " INTERACTION - Interagir avec quelqu'un

L'interaction est définie de la façon suivante : "Action réciproque de deux ou plusieurs phénomènes. "Il est également utilisé par les sociologues pour désigner les interactions sociales, c'est-à-dire les comportements d'action-réaction provoqués par la vie en société, ou plus simplement par la présence simultanée de deux individus au même endroit. 
2 Safer Internet Action Plan

3 Pour la partie belge francophone : Thierry De Smedt et Lysiane Romain, Internet et les jeunes, préface de Yves Thiran, éd. Média Animation, coll. Apprendre les médias, 2002.

Pour la partie française : Evelyne Bévort et Isabelle Bréda, Les jeunes et Internet. Représentations, usages et appropriations, CLEMI, Ministère de l'Education nationale, 2001.

4 Université de Sherbrooke (Jacques Piette et Christian-Marie Pons) et Université de Montréal (Luc Giroux).

5 L'Universidad de Granada et l'Universidad de Huelva pour l'Espagne ; le Centre de Liaison de l'Enseignement et des Moyens d'Information (CLEMI) pour la France ; l'Università cattolica del Sacro Cuore di Milano pour l'Italie ; l'Universidade de Coimbra pour le Portugal ; l'Université de Sherbrooke et l'Université de Montréal pour le Québec ; et le Centre d'éducation aux médias et aux technologies de l'information et de la communication (Cemtic) du canton de Vaud, pour la Suisse.

6 GRYSPEERDT Axel et KLEIN Annabelle, La galaxie des rumeurs, Bruxelles, Editions Vie Ouvrière, 1996

7 Thierry De Smedt et Lysiane Romain, Internet et les jeunes, préface de Yves Thiran, éd. Média Animation, coll. Apprendre les médias, 2002.

8 Evelyne Bévort et Isabelle Bréda, op. cit., pp.12-13.

9 Thierry De Smedt et Lysiane Romain, op. cit.

10 Lysiane Romain, Thierry De Smedt (dir.), Rapport d'observation finale d'Educaunet, GreMS - CLEMI - Média Animation, Internet Action Plan, Septembre 2002, Louvain-la-Neuve.

11 London Institute of Education - Centre for the study of children, youth and media, Andrew Burn (Lecturer), Rebekah Willett (Research Officer) University of London, Institute of Education Bedford Way 20 - WC1H OAL London

http://www.ccsonline.org.uk/mediacentre 